\title{
Analysis of peeling decoder for MET ensembles
}

\author{
Ryan W. Hinton \\ University of Virginia and L-3 Communications CSW \\ rwh4s@ virginia.edu
}

\author{
Stephen G. Wilson \\ University of Virginia \\ sgw@virginia.edu
}

\begin{abstract}
The peeling decoder introduced by Luby, et al. allows analysis of LDPC decoding for the binary erasure channel (BEC). For irregular ensembles, they analyze the decoder state as a Markov process and present a solution to the differential equations describing the process mean. Multi-edge type (MET) ensembles allow greater precision through specifying graph connectivity. We generalize the the peeling decoder for MET ensembles and derive analogous differential equations. We offer a new change of variables and solution to the node fraction evolutions in the general (MET) case. This result is preparatory to investigating finite-length ensemble behavior.
\end{abstract}

Index Terms-peeling decoder, multi-edge type (MET) ensembles, binary erasure channel (BEC), low-density parity-check (LDPC) codes

\section{INTRODUCTION}

Low-density parity-check codes (LDPC) offer excellent channel coding performance using a simple decoding algorithm [1]. Luby, et al. [2] introduced the peeling decoder as a tool for analyzing irregular ensembles over the binary erasure channel (BEC). With this tool they obtain a form of density evolution and conditions to determine threshold, the asymptotic limit of the ensemble decoding performance.

Multi-edge type (MET) LDPC ensembles [3] generalize irregular ensembles by allowing control over graph connectivity. In particular, the MET framework allows for degree-1 variable nodes, punctured variable nodes, and control over the graph structure that offer superior performance relative to irregular ensembles of comparable complexity (block length, average and maximum degree). In addition, MET ensembles are useful analytically as they include ensembles with interesting structure such as repeat-accumulate, irregular repeat-accumulate, and protograph codes as special cases - as well as regular and irregular ensembles.

In this paper we generalize the peeling decoder analysis to multi-edge type ensembles. Specifically, we present the modified difference equations and corresponding differential equations, introduce a new change of variables, and solve the system. The original MET analysis [3] treats belief propagation for the larger class of symmetric binary, memoryless, symmetric channels. However, the Markov chain setting is more easily applied to finite-length performance scaling [4]. We plan to build on this result to develop finite-length scaling laws for MET ensembles over the BEC and eventually for general channels as in [5].

This work was supported in part by the National Science Foundation under grant number ECCS 0636598.
The remainder of the paper is organized as follows. Section II provides background on and notation for the peeling decoder and multi-edge type ensembles. Section III describes the system of difference and ordinary differential equations (ODEs) for the peeling decoder applied to MET ensembles. Section IV presents a new change of variables and the corresponding ODE system solution. Section $\mathrm{V}$ discusses this solution and concludes the paper.

\section{BACKGROUND}

\section{A. Multi-edge type ensembles}

An MET ensemble [3] $\operatorname{LDPC}(N, \nu, \mu)$ is specified by the transmitted block length $N$ and a pair of multivariate generating functions, $\nu(\mathbf{r}, \mathbf{x})$ and $\mu(\mathbf{x})$

$$
\begin{aligned}
\nu(\mathbf{r}, \mathbf{x}) & =\sum_{\mathbf{b}, \mathbf{d}} \nu_{\mathbf{b}, \mathbf{d}} \mathbf{r}^{\mathbf{b}} \mathbf{x}^{\mathbf{d}} & \mu(\mathbf{x}) & =\sum_{\mathbf{d}} \mu_{\mathbf{d}} \mathbf{x}^{\mathbf{d}} \\
\mathbf{d} & =\left(d_{1}, d_{2}, \ldots, d_{n_{\mathfrak{e}}}\right) & \mathbf{b} & =\left(b_{0}, b_{1}, \ldots, b_{n_{\mathfrak{r}}}\right) \\
\mathbf{x} & =\left(x_{1}, x_{2}, \ldots, x_{n_{\mathfrak{e}}}\right) & \mathbf{r} & =\left(r_{0}, r_{1}, \ldots, r_{n_{\mathfrak{r}}}\right)
\end{aligned}
$$

where $\mathbf{x}^{\mathbf{d}}=\prod_{i=1}^{n_{\mathfrak{e}}} x_{i}^{d_{i}}$ and $\mathbf{r}^{\mathbf{b}}=\prod_{i=0}^{n_{\mathfrak{r}}} r_{i}^{b_{i}}$. Note that the edge type index (i.e. the subscript $i$ on $x_{i}$ ) goes from 1 to $n_{\mathfrak{e}}$ while the channel type index (i.e. subscripts of $r_{i}$ ) goes from 0 to $n_{\mathfrak{r}}$ in order to include the punctured channeli $r_{0}$. Each term $\left(\nu_{\mathbf{b}, \mathbf{d}} \mathbf{r}^{\mathbf{b}} \mathbf{x}^{\mathbf{d}}\right)$ in $\nu$ corresponds to variable nodes of a single type: the coefficient $\nu_{\mathbf{b}, \mathbf{d}}$ is the fraction of nodes of type $(\mathbf{b}, \mathbf{d})$, the exponent $\left(b_{i}\right)$ on each channel type variable $\left(r_{i}\right)$ indicates the number of connections to that channe ${ }^{\text {ii }}$, and the exponent $\left(d_{j}\right)$ on each edge type variable $\left(x_{j}\right)$ indicates the number of sockets of that type. The corresponding definitions hold for the coefficients $\mu_{\mathbf{d}}$ and edge type exponents $\mathbf{d}$ of the terms of $\mu$. In particular, note that variable nodes have type or degree $(\mathbf{b}, \mathbf{d})$ to distinguish their channel and edge connectivity while check nodes are distinguished solely by their edge connectivity, d. As with irregular ensembles, the ensemble consists of the codes corresponding to every (compatible) socket permutation.

The remaining ensemble characteristics can be derived from these polynomials. The number of edges of type $i$ connected to a particular variable node or check node type is given by $N d_{i} \nu_{\mathbf{b}, \mathbf{d}}$ and $N d_{i} \mu_{\mathbf{d}}$, respectively. Define

$$
\nu_{x_{i}}(\mathbf{r}, \mathbf{x})=\frac{\partial}{\partial x_{i}} \nu(\mathbf{r}, \mathbf{x}) \quad \mu_{x_{i}}(\mathbf{r}, \mathbf{x})=\frac{\partial}{\partial x_{i}} \mu(\mathbf{r}, \mathbf{x})
$$

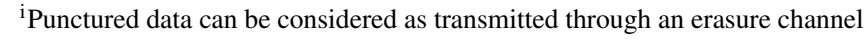
with erasure probability 1 .

${ }^{\text {ii }}$ The channel type exponents $\mathbf{b}$ typically have a single non-zero entry of 1 indicating that each bit receives exactly one channel realization. 

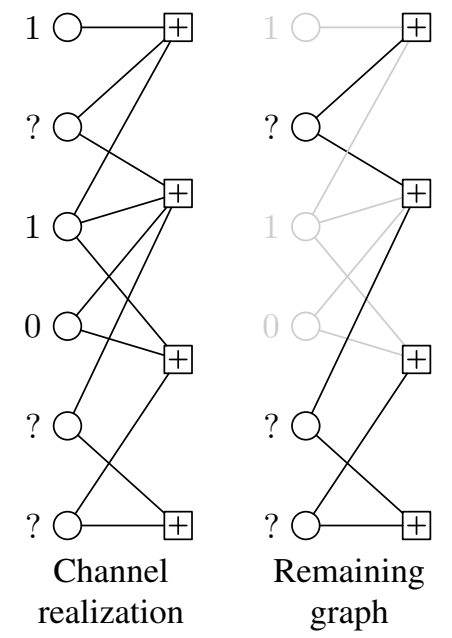

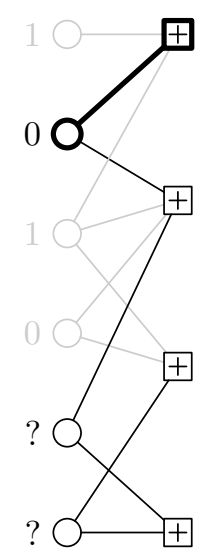

Iteration 1

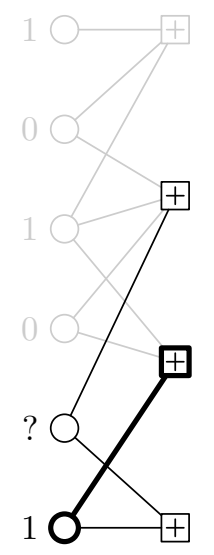

Iteration 2

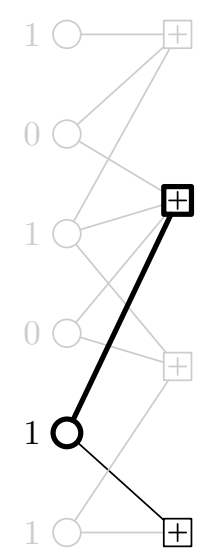

Iteration 3

Fig. 1. Peeling decoder operation.

and the total number of edges of type $i$ as

$$
E_{i}=N \nu_{x_{i}}(\mathbf{1}, \mathbf{1})=N \mu_{x_{i}}(\mathbf{1})
$$

and $E=\sum_{i} E_{i}$ the total number of edges. The edgeperspective degree profiles are

$$
\begin{gathered}
\boldsymbol{\lambda}(\mathbf{r}, \mathbf{x})=\left(\frac{\nu_{x_{1}}(\mathbf{r}, \mathbf{x})}{\nu_{x_{1}}(\mathbf{1}, \mathbf{1})}, \ldots, \frac{\nu_{x_{n_{\mathfrak{e}}}}(\mathbf{r}, \mathbf{x})}{\nu_{x_{n_{\mathfrak{e}}}}(\mathbf{1}, \mathbf{1})}\right) \\
\boldsymbol{\rho}(\mathbf{x})=\left(\frac{\mu_{x_{1}}(\mathbf{x})}{\mu_{x_{1}}(\mathbf{1})}, \ldots, \frac{\mu_{x_{n_{\mathfrak{e}}}}(\mathbf{x})}{\mu_{x_{n_{\mathfrak{e}}}}(\mathbf{1})}\right)
\end{gathered}
$$

where the denominators can also expressed as $E_{i} / N$.

\section{B. Peeling decoder}

The peeling decoder of [2] attempts to recover an LDPC code word transmitted over the erasure channel. Its operation is depicted in Fig. 1. Given the channel realization (i.e. which bits were erased by the channel), each check node sums the known values from its attached variable nodes. Then the known variable nodes and their edges are deleted from the decoding (Tanner) graph since they convey no further information. The check node constraint allows us to deduce the value of a variable node connected to a check node with degree 1 in the remaining graph. Decoding proceeds iteratively on this principle. At each iteration $T=1,2, \ldots$, a degree- 1 check node is selected at random. The attached variable node value is computed, then this node and the selected check node are deleted along with their edges. Decoding stops when no degree-1 check nodes remain. If the remaining graph is empty, decoding was successful. Otherwise, the remaining variable nodes form a stopping set [6], [7]. The critical insight is that the distribution of socket permutations for the remaining graph at each iteration remains uniform, so the evolution of the degree sequence can be described as a Markov chain of degree distributions.

\section{PeEling DeCoder FOR MET ENSEMbles}

Consider the operation of the peeling decoder on a code from an MET ensemble. This development closely follows [2].
However, we examine the evolution of node fractions instead of edge fractions: allowing multiple edge types results in a proliferation of redundant edge-perspective equations.

Analyzing the peeling decoder for MET ensembles requires extending and adding notation. The node fractions are normalized by $N$ while the edge fractions are normalized by $E$, so the average variable node degree $\bar{d}_{v}=E / N$ and per-edge type average degrees $\bar{d}_{v, i}=E_{i} / N$ frequently appear as conversion factors. Let $\mathbf{e}_{i}$ denote the unit vector with a 1 in position $i$ and zeros elsewhere. By considering only the erasure channel, the vector of erasure probabilities $\epsilon=\left(1, \epsilon_{1}, \epsilon_{2}, \ldots\right)$ can be substituted for the general distributions $\mathbf{r}$.

Consider the decoder state at time $t=T / E$. Define the time functions $\nu_{\mathbf{b}, \mathbf{d}}(t)$ to indicate the remaining fraction (relative to $N$ ) of variable nodes of each type $(\mathbf{b}, \mathbf{d})$ at time $t$ and similarly for check nodes. These time-varying parameters are not to be confused with $\nu_{\mathbf{b}, \mathbf{d}}$ and $\mu_{\mathbf{d}}$, the fraction of nodes of each type in the Tanner graph before decoding. Denote $\ell_{i, \mathbf{b}, \mathbf{d}}(t)=$ $\bar{d}_{v} d_{i} \nu_{\mathbf{b}, \mathbf{d}}(t)$ the remaining fraction of edges (relative to $E$ ) of type $i$ connected to variable nodes of type $(\mathbf{b}, \mathbf{d})$ at time $t$ and $r_{i, \mathbf{d}}(t)=\bar{d}_{v} d_{i} \mu_{\mathbf{d}}(t)$ the analogous check node edge fraction. Summing either set gives

$$
e_{i}(t)=\sum_{\mathbf{b}, \mathbf{d}} \ell_{i, \mathbf{b}, \mathbf{d}}(t)=\sum_{\mathbf{d}} r_{i, \mathbf{d}}(t),
$$

the remaining fraction of edges of type $i$ at time $t$. Denote $N_{\mathbf{b}, \mathbf{d}}(t)=N \nu_{\mathbf{b}, \mathbf{d}}(t)$ the expected number of variable nodes of type $(\mathbf{b}, \mathbf{d})$ at time $t$ and $M_{\mathbf{d}}(t)=N \mu_{\mathbf{d}}(t)$ the analogous check node mean. Let the time step $\Delta t=1 / E$. Finally, denote the indicator function

$$
I\{x\}= \begin{cases}1 & \text { if } x \text { is true } \\ 0 & \text { otherwise }\end{cases}
$$

At each successful iteration, the peeling decoder deletes a check node of degree one, attached variable node, and their edges. For a code from a multi-edge type ensemble, there may be degree-one check nodes of several types from which to choose. Define the time-indexed sequence of random 
variables $\Gamma(t)$ whose pmfs $\gamma_{i}(t)=\mathrm{P}(\Gamma(t)=i)$ indicate the probability of choosing a degree- $\mathbf{e}_{i}$ check node at each iteration. Interestingly, $\Gamma(t)$ presents a free variable for the decoder of $n_{\mathfrak{e}}-1$ dimensions at each iteration. Choosing $\Gamma(t)$ is discussed in Section $\mathrm{V}$. Assuming the decoder chooses a degree-1 check node $(\mathrm{CN})$ of type $i$, the probability that the attached variable node $(\mathrm{VN})$ has type $(\mathbf{b}, \mathbf{d})$ is

$$
\frac{\ell_{i, \mathbf{b}, \mathbf{d}}(t)}{e_{i}(t)}=\frac{\bar{d}_{v} d_{i} \nu_{\mathbf{b}, \mathbf{d}}(t)}{e_{i}(t)} .
$$

This value times $\gamma_{i}(t)$ gives the (joint) probability of choosing an edge of type $i$ attached to a variable node of type $(\mathbf{b}, \mathbf{d})$. Hence the marginal

$$
\sum_{i=1}^{n_{\mathrm{e}}} \gamma_{i}(t) \frac{\ell_{i, \mathbf{b}, \mathbf{d}}(t)}{e_{i}(t)}=\frac{1}{\bar{d}_{v}} \nu_{\mathbf{b}, \mathbf{d}}(t) \sum_{i} \frac{d_{i} \gamma_{i}(t)}{e_{i}(t)}
$$

gives the total probability of removing a variable node of type $(\mathbf{b}, \mathbf{d})$ at time $t$. The difference in the expected number of nodes of each type is

$$
N_{\mathbf{b}, \mathbf{d}}(t+\Delta t)-N_{\mathbf{b}, \mathbf{d}}(t)=-\frac{1}{\bar{d}_{v}} \nu_{\mathbf{b}, \mathbf{d}}(t) \sum_{i} \frac{d_{i} \gamma_{i}(t)}{e_{i}(t)} .
$$

Having selected a degree- $\mathbf{e}_{i}$ check node attached to a variable node of type $(\mathbf{b}, \mathbf{d})$, the decoder removes these nodes and their edges from the graph. Removing the variable node's edges changes the degree or type of the connected check nodes. One edge connects to the original degree-1 check node, but we need to account for the other $\mathbf{d}-\mathbf{e}_{i}$ edges. The expected number of "other" edges of type $j$ deleted is iii

$$
\begin{aligned}
& \sum_{i, \mathbf{b}, \mathbf{d}}\left(d_{j}-I\{i=j\}\right) \gamma_{i}(t) \frac{\ell_{i, \mathbf{b}, \mathbf{d}}(t)}{e_{i}(t)} \\
& =\left[\frac{1}{\bar{d}_{v}} \sum_{\mathbf{b}, \mathbf{d}} d_{j} \nu_{\mathbf{b}, \mathbf{d}}(t) \sum_{i} \frac{d_{i} \gamma_{i}(t)}{e_{i}(t)}\right]-\left[\frac{\gamma_{j}(t)}{e_{j}(t)} \cdot \sum_{\mathbf{b}, \mathbf{d}} \ell_{j, \mathbf{b}, \mathbf{d}}(t)\right] \\
& =\tilde{a}_{j}(t)-\gamma_{j}(t)
\end{aligned}
$$

where

$$
\tilde{a}_{j}(t)=\frac{1}{\bar{d}_{v}} \sum_{\mathbf{b}, \mathbf{d}} d_{j} \nu_{\mathbf{b}, \mathbf{d}}(t) \sum_{i} \frac{d_{i} \gamma_{i}(t)}{e_{i}(t)}
$$

is the expected (total) number of edges of type $j$ deleted at time $t$. Assume each "other" edge is connected to an unique check node since the computation graph is tree-like in the large block length limit. The probability that a deleted variable node edge of type $j$ is connected to a check node of type $\mathbf{d}$ is $r_{j, \mathbf{d}}(t) / e_{j}(t)=d_{j} \mu_{\mathbf{d}}(t) /\left[\bar{d}_{v} e_{j}(t)\right]$. Deleting an edge of type $j$ from a check node of type $\mathbf{d}+\mathbf{e}_{j}$ removes one node of this type and adds a node of type $\mathbf{d}$. So the expected number of added check nodes of type $\mathbf{d}$ is

$$
\sum_{j}\left[\tilde{a}_{j}(t)-\gamma_{j}(t)\right] \frac{\left(d_{j}+1\right) \mu_{\mathbf{d}+\mathbf{e}_{j}}(t)}{\bar{d}_{v} e_{j}(t)}
$$

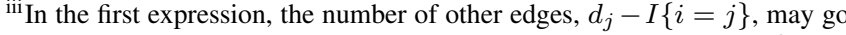
negative. However, this is not a concern since this only happens when $d_{j}=0$ so $\ell_{i, \mathbf{b}, \mathbf{d}}(t)$ is zero.
}

and the expected number of removed check nodes of type $\mathbf{d}$ is

$$
\gamma_{i}(t) I\{\mathbf{d} \text { is degree- } 1\}+\sum_{j}\left[\tilde{a}_{j}(t)-\gamma_{j}(t)\right] \frac{d_{j} \mu_{\mathbf{d}}(t)}{\bar{d}_{v} e_{j}(t)} .
$$

The expected change in the number of check nodes of type $\mathbf{d}$ is simply their difference,

$$
\begin{aligned}
& M_{\mathbf{d}}(t+\Delta t)-M_{\mathbf{d}}(t) \\
& =\sum_{j}\left[\left(d_{j}+1\right) \mu_{\mathbf{d}+\mathbf{e}_{j}}(t)-d_{j} \mu_{\mathbf{d}}(t)\right] \frac{\tilde{a}_{j}(t)-\gamma_{j}(t)}{\bar{d}_{v} e_{j}(t)} \\
& \quad-\sum_{i} \gamma_{i}(t) I\left\{\mathbf{d}=\mathbf{e}_{i}\right\} .
\end{aligned}
$$

The system of difference equations given by (1) and (3) describe the evolution of the expected number of variable and check nodes of each type. These quantities also provide the transition probabilities for the Markov chain whose state is these node counts. Since the time axis $t$ is scaled inversely with $E$, in the large block length limit $\Delta t \rightarrow 0$ and the discrete-time Markov chain approaches a continuous-time Markov process (see [4] for a formal description). The transition rates for this process are the limits of the corresponding difference equations. Since $N_{\mathbf{b}, \mathbf{d}}(t)=N \nu_{\mathbf{b}, \mathbf{d}}(t)=E \nu_{\mathbf{b}, \mathbf{d}}(t) / \bar{d}_{v}=$ $\nu_{\mathbf{b}, \mathbf{d}} /\left(\bar{d}_{v} \Delta t\right)$, the limiting variable node rates are

$$
\frac{\mathrm{d}}{\mathrm{d} t} \nu_{\mathbf{b}, \mathbf{d}}(t)=-\nu_{\mathbf{b}, \mathbf{d}}(t) \sum_{i} \frac{d_{i} \gamma_{i}(t)}{e_{i}(t)} .
$$

Similarly, the check node fractions follow

$$
\begin{gathered}
\frac{\mathrm{d}}{\mathrm{d} t} \mu_{\mathbf{d}}(t)=\sum_{j}\left[\left(d_{j}+1\right) \mu_{\mathbf{d}+\mathbf{e}_{j}}(t)-d_{j} \mu_{\mathbf{d}}(t)\right] \frac{\tilde{a}_{j}(t)-\gamma_{j}(t)}{e_{j}(t)} \\
-\bar{d}_{v} \sum_{i} \gamma_{i}(t) I\left\{\mathbf{d}=\mathbf{e}_{i}\right\}
\end{gathered}
$$

\section{A. Initial conditions}

A complete solution requires the process initial conditions as well. The known nodes are deleted after the channel realization, so variable nodes attached to channel type $k$ are retained with probability $\epsilon_{k}$. Accordingly, the initial means are

$$
\nu_{\mathbf{b}, \mathbf{d}}(0)=\epsilon^{\mathbf{b}} \nu_{\mathbf{b}, \mathbf{d}} .
$$

In other words, each variable node is deleted from the remaining graph according to the probability that it is not erased over its attached channel.

The check node fractions are more interesting. The probability that an edge of type $i$ is retained before the first iteration is

$$
\frac{\sum_{\mathbf{b}, \mathbf{d}} d_{i} \boldsymbol{\epsilon}^{\mathbf{b}} \nu_{\mathbf{b}, \mathbf{d}}}{\sum_{\mathbf{b}, \mathbf{d}} d_{i} \nu_{\mathbf{b}, \mathbf{d}}}=\frac{\sum_{\mathbf{b}, \mathbf{d}} d_{i} \nu_{\mathbf{b}, \mathbf{d}}(0)}{E_{i} / N}=\lambda_{i}(\boldsymbol{\epsilon}, \mathbf{1}),
$$

the weighted average of the probabilities that the variable nodes attached to this edge type will be retained. A check 
node type may be produced by deleting edges from any initial check node type with larger degree, so

$$
\mu_{\mathbf{d}}(0)=\sum_{\tilde{\mathbf{d}} \geq \mathbf{d}} \mu_{\tilde{\mathbf{d}}}\left(\begin{array}{l}
\tilde{\mathbf{d}} \\
\mathbf{d}
\end{array}\right) \boldsymbol{\lambda}(\boldsymbol{\epsilon}, \mathbf{1})^{\mathbf{d}}[\mathbf{1}-\boldsymbol{\lambda}(\boldsymbol{\epsilon}, \mathbf{1})]^{\tilde{\mathbf{d}}-\mathbf{d}}
$$

where $\tilde{\mathbf{d}} \geq \mathbf{d}$ when $\tilde{d}_{i} \geq d_{i}$ for $i=1, \ldots, n_{\mathfrak{e}}$ and the vector binomial denotes the product of binomial coefficients,

$$
\left(\begin{array}{l}
\tilde{\mathbf{d}} \\
\mathbf{d}
\end{array}\right)=\prod_{i}\left(\begin{array}{l}
\tilde{d}_{i} \\
d_{i}
\end{array}\right) \text {. }
$$

Note that the only non-zero terms in (7) are those with positive fractions in the original Tanner graph, $\left\{\tilde{\mathbf{d}}: \mu_{\tilde{\mathbf{d}}}>0\right\}$.

\section{SOlution FOR MET PEELING DECODER EVOLUTION}

The ODE system solution for multi-edge types is similar to that for the single edge case. One key novelty is the change of variables from $t$ to $\mathbf{x}=\left(x_{1}, x_{2}, \ldots, x_{n_{\mathrm{e}}}\right)$ with $x_{i}$ defined implicitly by

$$
\frac{\gamma_{i}(t)}{e_{i}(t)}=-\frac{1}{x_{i}} \cdot \frac{\mathrm{d} x_{i}}{\mathrm{~d} t}
$$

This substitution conflates time with choice of $\Gamma(t)$. For clarity, we will refer to $\mathbf{x}$ and its elements without making either dependence explicit. Using this substitution, the variable node differential equation is

$$
\frac{\mathrm{d}}{\mathrm{d} t} \nu_{\mathbf{b}, \mathbf{d}}(\boldsymbol{\epsilon}, \mathbf{x})=\nu_{\mathbf{b}, \mathbf{d}}(\boldsymbol{\epsilon}, \mathbf{x}) \sum_{i} \frac{d_{i}}{x_{i}} \cdot \frac{\mathrm{d} x_{i}}{\mathrm{~d} t}
$$

with solution

$$
\nu_{\mathbf{b}, \mathbf{d}}(\boldsymbol{\epsilon}, \mathbf{x})=\nu_{\mathbf{b}, \mathbf{d}} \boldsymbol{\epsilon}^{\mathbf{b}} \mathbf{x}^{\mathbf{d}}
$$

including the initial condition $(6)$ at $\mathbf{x}=\mathbf{1}$.

The more involved check node derivation is presented in Appendix [ but we include the result for degree- 1 check nodes here.

Lemma 1. The solution to the system (5), (7) for degree-1 check nodes is given by

$$
\mu_{\mathbf{e}_{i}}(\boldsymbol{\epsilon}, \mathbf{x})=\nu_{x_{i}}(\boldsymbol{\epsilon}, \mathbf{x})\left[x_{i}-1+\rho_{i}(\mathbf{1}-\boldsymbol{\lambda}(\boldsymbol{\epsilon}, \mathbf{x}))\right] .
$$

Note that this solution is equivalent to the the result from [2] for the single-edge case.

\section{DisCUSSION OF SOLUTION AND X-SPACE PATH}

Equation (10) provides the fraction of degree-1 check nodes of each type for any choice of schedule, $\Gamma(\cdot)$. The node fraction solutions are defined over the entire space $\mathcal{X}=$ $[0,1]^{n_{\mathfrak{e}}}$, but the decoder behavior is modeled by these equations evaluated over the path $\mathbf{x}(t)$ induced by the schedule. In particular, the natural choice is for the peeling decoder to select a degree-1 check node without respect to edge type, i.e. according to the proportion of remaining degree- 1 nodes of that type:

$$
\gamma_{i}(\boldsymbol{\epsilon}, \mathbf{x})=\frac{\mu_{\mathbf{e}_{i}}(\boldsymbol{\epsilon}, \mathbf{x})}{\sum_{j} \mu_{\mathbf{e}_{j}}(\boldsymbol{\epsilon}, \mathbf{x})}
$$

This choice yields another system of differential equations for $\mathbf{x}(t)$ which we have not yet solved.

For an ensemble with a single channel type (besides perhaps the punctured channel), define the schedule threshold for a particular schedule as

$\epsilon_{\Gamma}^{*}=\sup \left\{\epsilon: \mu_{\mathbf{e}_{i}}(\mathbf{x}(t))>0 \forall i=1, \ldots, n_{\mathfrak{e}}\right.$ and $\left.t \in\left[0, t_{f}\right)\right\}$

where $\mathbf{x}(t)$ denotes the path induced by the schedule $\Gamma$ and $t_{f}=\nu(\boldsymbol{\epsilon}, \mathbf{1}) / \bar{d}_{v}$ is the expected decoder completion time. Also define the ensemble threshold as

$$
\epsilon^{*}=\max _{\Gamma}\left\{\epsilon_{\Gamma}^{*}\right\} .
$$

In fact, the choice of schedule is not critical. A reasonable schedule always chooses an edge type with a positive fraction of degree-1 check nodes when one is available. For example, choosing $\gamma_{i}(t)=I\{i=0\} 1$ is an unreasonable schedule for a two-edge type code since the decoder will fail when the supply

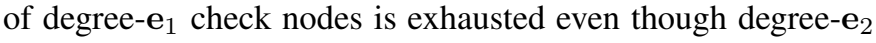
check nodes may be available.

Lemma 2. Every reasonable schedule $\Gamma$ has $\epsilon_{\Gamma}^{*}=\epsilon^{*}$. In other words, every reasonable schedule achieves the ensemble threshold.

Proof: Failure events on the BEC are given by stopping sets [7]. A reasonable schedule will fail only at a stopping set. Since no schedule can decode past the unique maximal stopping set, every reasonable schedule must yield the same (optimal) error pattern.

By standard arguments [2], the behavior of codes from an MET ensemble concentrate in probability around the means (9), (10) and (16) as the block lengh increases. For $\epsilon<\epsilon^{*}$, all of the degree-1 check node fractions remain positive with high probability until decoding succeeds $\left(t=t_{f}\right)$. Likewise for $\epsilon>\epsilon^{*}$, the decoder fails with high probability.

Below threshold (high probability of success), the differential equations for $\mathbf{x}(t)$ can be integrated numerically as shown in Fig. 2. Above threshold, all the degree-1 check node fractions go to zero for some $\mathbf{x}(t)$, so the natural schedule probabilities $\gamma_{i}$ (and hence the equations for $\mathbf{x}$ ) are indeterminate $(0 / 0)$.

\section{APPENDIX}

In this section we present a derivation of the solution to the differential equations describing the mean decoding trajectory for the peeling decoder operating on an MET ensemble. In the sequel, we denote $\gamma_{i}$ as a function of $\mathbf{x}$ (time) and $\boldsymbol{\epsilon}$ in accordance with Section $\mathrm{V}$ where the choice of $\Gamma$ depends on time (through $\mathbf{x}$ ) and $\epsilon$. Consider the fraction of check nodes of degree greater than one. Denoting

$$
\lambda_{j}^{\prime}(\boldsymbol{\epsilon}, \mathbf{x})=\frac{\mathrm{d}}{\mathrm{d} t} \lambda_{j}(\boldsymbol{\epsilon}, \mathbf{x})=\sum_{i} \frac{\mathrm{d} x_{i}}{\mathrm{~d} t} \frac{\partial}{\partial x_{i}} \lambda_{j}(\boldsymbol{\epsilon}, \mathbf{x}),
$$

the expected number of "other" edges deleted simplifies to

$$
\tilde{a}_{j}(t)=-\frac{E_{j}}{E} x_{j} \lambda_{j}^{\prime}(\boldsymbol{\epsilon}, \mathbf{x}) .
$$



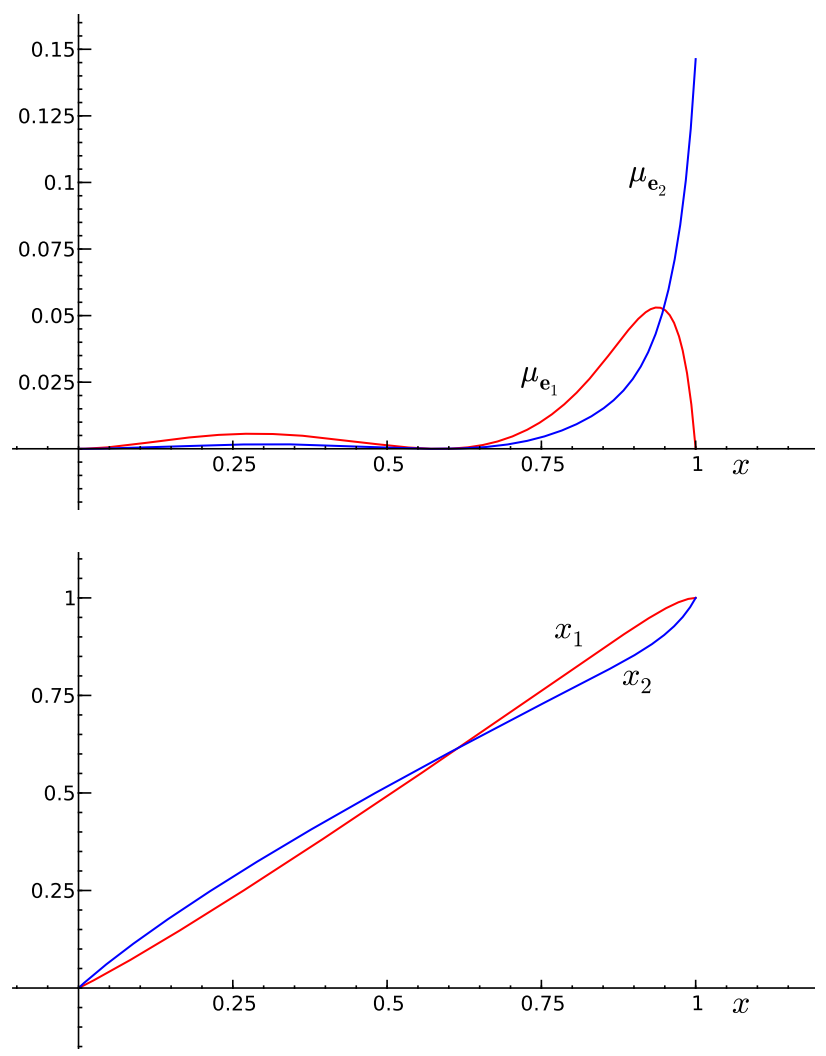

Fig. 2. Example of peeling decoder state evolution for repeat-accumulate ensemble, systematic bits punctured, rate 1/3. From [3], this ensemble has two edge types, $\nu(\mathbf{r}, \mathbf{x})=r_{1} x_{1}^{2}+\frac{1}{3} r_{0} x_{2}^{3}$ and $\mu(\mathbf{x})=x_{1}^{2} x_{2}$. These plots follow the natural path for $\epsilon=0.6175 \approx \epsilon^{*}$. The horizontal axis for both plots is $x=\sum_{i} x_{i} E_{i} / E$, the weighted average of the elements of $\mathbf{x}$.

From the variable node solutions, the edge type fractions are

$$
e_{i}(\boldsymbol{\epsilon}, \mathbf{x})=\frac{E_{i}}{E} x_{i} \lambda_{i}(\boldsymbol{\epsilon}, \mathbf{x}) .
$$

Substituting these expressions into [5], the check node fractions follow

$$
\begin{aligned}
& \frac{\mathrm{d}}{\mathrm{d} t} \mu_{\mathbf{d}}(\boldsymbol{\epsilon}, \mathbf{x}) \\
= & \sum_{j}\left[-\left(d_{j}+1\right) \mu_{\mathbf{d}+\mathbf{e}_{j}}(\boldsymbol{\epsilon}, \mathbf{x})+d_{j} \mu_{\mathbf{d}}(\boldsymbol{\epsilon}, \mathbf{x})\right] \frac{\lambda_{j}^{\prime}(\boldsymbol{\epsilon}, \mathbf{x})}{\lambda_{j}(\boldsymbol{\epsilon}, \mathbf{x})} .
\end{aligned}
$$

Similar to [2], the "explicit" solutions to (13) are

$$
\begin{aligned}
& \mu_{\mathbf{d}}(\boldsymbol{\epsilon}, \mathbf{x})=\boldsymbol{\lambda}(\boldsymbol{\epsilon}, \mathbf{x})^{\mathbf{d}}\left[c_{\mathbf{d}}\right. \\
& \left.-\sum_{j}\left(d_{j}+1\right) \int_{\tau=0}^{t} \mu_{\mathbf{d}+\mathbf{e}_{j}}(\boldsymbol{\epsilon}, \mathbf{x}) \frac{1}{\boldsymbol{\lambda}(\boldsymbol{\epsilon}, \mathbf{x})^{\mathbf{d}}} \frac{\lambda_{j}^{\prime}(\boldsymbol{\epsilon}, \mathbf{x})}{\lambda_{j}(\boldsymbol{\epsilon}, \mathbf{x})} \mathrm{d} \tau\right]
\end{aligned}
$$

(inside the integral $\mathbf{x}$ is a function of $\tau$ in place of $t$ ) which admit the solutions

$$
\mu_{\mathbf{d}}(\boldsymbol{\epsilon}, \mathbf{x})=\sum_{\tilde{\mathbf{d}} \geq \mathbf{d}}(-1)^{\|\tilde{\mathbf{d}}-\mathbf{d}\|}\left(\begin{array}{l}
\tilde{\mathbf{d}} \\
\mathbf{d}
\end{array}\right) c_{\tilde{\mathbf{d}}} \boldsymbol{\lambda}(\boldsymbol{\epsilon}, \mathbf{x})^{\tilde{\mathbf{d}}} .
$$

where $\|\mathbf{d}\|=\sum_{i} d_{i}$ is the $L_{1}$ norm. Note that this sum ranges over all larger check node degrees with non-zero time-dependent fractions. The constants $\left\{c_{\mathbf{d}}\right\}$ are determined recursively from the initial conditions as

$$
\mu_{\mathbf{d}}(\boldsymbol{\epsilon}, \mathbf{x})=\sum_{\tilde{\mathbf{d}} \geq \mathbf{d}} \mu_{\tilde{\mathbf{d}}}\left(\begin{array}{l}
\tilde{\mathbf{d}} \\
\mathbf{d}
\end{array}\right) \boldsymbol{\lambda}(\boldsymbol{\epsilon}, \mathbf{x})^{\mathbf{d}}[\mathbf{1}-\boldsymbol{\lambda}(\boldsymbol{\epsilon}, \mathbf{x})]^{\tilde{\mathbf{d}}-\mathbf{d}}
$$

for check nodes of degree greater than one. This solution obviously satisfies the initial conditions (7), and it can be shown that it also satisfies (13).

The fraction of degree- 1 check nodes is the difference between the total fraction of type- $i$ edges and the fraction of type- $i$ edges attached to higher-degree check nodes.

$$
\begin{aligned}
\mu_{\mathbf{e}_{i}}(\boldsymbol{\epsilon}, \mathbf{x}) & =\bar{d}_{v} e_{i}(\boldsymbol{\epsilon}, \mathbf{x})-\sum_{\mathbf{d} \neq \mathbf{e}_{i}} d_{i} \mu_{\mathbf{d}}(\boldsymbol{\epsilon}, \mathbf{x}) \\
= & \bar{d}_{v} e_{i}(\boldsymbol{\epsilon}, \mathbf{x}) \\
& -\sum_{\mathbf{d} \neq \mathbf{e}_{i}} d_{i} \sum_{\tilde{\mathbf{d}} \geq \mathbf{d}} \mu_{\tilde{\mathbf{d}}}\left(\begin{array}{c}
\tilde{\mathbf{d}} \\
\mathbf{d}
\end{array}\right) \boldsymbol{\lambda}(\boldsymbol{\epsilon}, \mathbf{x})^{\mathbf{d}}[\mathbf{1}-\boldsymbol{\lambda}(\boldsymbol{\epsilon}, \mathbf{x})]^{\tilde{\mathbf{d}}-\mathbf{d}} \\
= & \bar{d}_{v} e_{i}(\boldsymbol{\epsilon}, \mathbf{x}) \\
& -\sum_{\tilde{\mathbf{d}}} \mu_{\tilde{\mathbf{d}}} \sum_{\mathbf{d} \leq \tilde{\mathbf{d}}} d_{i}\left(\begin{array}{c}
\tilde{\mathbf{d}} \\
\mathbf{d}
\end{array}\right) \boldsymbol{\lambda}(\boldsymbol{\epsilon}, \mathbf{x})^{\mathbf{d}}[\mathbf{1}-\boldsymbol{\lambda}(\boldsymbol{\epsilon}, \mathbf{x})]^{\tilde{\mathbf{d}}-\mathbf{d}} \\
& +\sum_{\tilde{\mathbf{d}} \geq \mathbf{e}_{i}} \mu_{\tilde{\mathbf{d}}}\left(\begin{array}{c}
\tilde{\mathbf{d}} \\
\mathbf{e}_{i}
\end{array}\right) \boldsymbol{\lambda}(\boldsymbol{\epsilon}, \mathbf{x})^{\mathbf{e}_{i}}[\mathbf{1}-\boldsymbol{\lambda}(\boldsymbol{\epsilon}, \mathbf{x})]^{\tilde{\mathbf{d}}-\mathbf{e}_{i}}
\end{aligned}
$$

From definitions, the third term simplifies to $\bar{d}_{v, i} \lambda_{i}(\boldsymbol{\epsilon}, \mathbf{x}) \rho_{i}(\mathbf{1}-\boldsymbol{\lambda}(\boldsymbol{\epsilon}, \mathbf{x}))$. Furthermore, the second term is equal to $\sum_{\tilde{\mathbf{d}}} \mu_{\tilde{\mathbf{d}}} \tilde{d}_{i} \lambda_{i}(\boldsymbol{\epsilon}, \mathbf{x})=\bar{d}_{v, i} \lambda_{i}(\boldsymbol{\epsilon}, \mathbf{x})=\nu_{i}(\boldsymbol{\epsilon}, \mathbf{x})$ iv Combining these simplifications with $\bar{d}_{v} e_{i}(\epsilon, \mathbf{x})=$ $\bar{d}_{v, i} x_{i} \lambda_{i}(\boldsymbol{\epsilon}, \mathbf{x})$, we have the solution (10) given in Lemma 1

\section{REFERENCES}

[1] S.-Y. Chung, G. D. Forney, T. J. Richardson, and R. L. Urbanke, "On the design of low-density parity check codes within $0.0045 \mathrm{~dB}$ of the Shannon limit," IEEE Commun. Lett., vol. 5, no. 2, pp. 58-60, Feb. 2001.

[2] M. G. Luby, M. Mitzenmacher, M. A. Shokrollahi, D. A. Spielman, and V. Stemann, "Practical loss-resilient codes," Proc. ACM Symp. on Theory of Computing, pp. 150-159, 1997.

[3] T. J. Richardson and R. L. Urbanke, "Multi-edge type LDPC codes," May 24-25 2002, presented at the Workshop honoring Prof. Bob McEliece on his 60th birthday.

[4] A. Amraoui, A. Montanari, T. Richardson, and R. Urbanke, "Finitelength scaling for iteratively decoded LDPC ensembles," IEEE Trans. Inf. Theory, vol. 55, no. 2, pp. 473-498, Feb. 2009.

[5] J. Ezri, A. Montanari, and R. Urbanke, "A generalization of the finitelength scaling approach beyond the BEC," in Proc. 2007 Int. Symp. Information Theory, 2007, pp. 1011-1015.

[6] T. J. Richardson and R. L. Urbanke, "Efficient encoding of low-density parity-check codes," IEEE Trans. Inf. Theory, vol. 47, no. 2, pp. 638-656, Feb. 2001.

[7] C. Di, D. Proietti, I. E. Telatar, T. J. Richardson, and R. L. Urbanke, "Finite-length analysis of low-density parity-check codes on the binary erasure channel," IEEE Trans. Inf. Theory, vol. 48, no. 6, Jun. 2002.

\footnotetext{
${ }^{\text {iv }}$ Compare the generating functions $(\mathbf{a}+\mathbf{b})^{\tilde{\mathbf{d}}}$ and $a_{i} \frac{\partial}{\partial a_{i}}(\mathbf{a}+\mathbf{b})^{\tilde{\mathbf{d}}}$ and
} their expansions with $\mathbf{a}=\boldsymbol{\lambda}(\boldsymbol{\epsilon}, \mathbf{x})=\mathbf{1}-\mathbf{b}$. 